Bull. Korean Math. Soc. 52 (2015), No. 5, pp. 1433-1443

http://dx.doi.org/10.4134/BKMS.2015.52.5.1433

\title{
CIRCLE-FOLIATED MINIMAL SURFACES IN 4-DIMENSIONAL SPACE FORMS
}

\author{
SUNG-Ho PARK
}

\begin{abstract}
Catenoid and Riemann's minimal surface are foliated by circles, that is, they are union of circles. In $\mathbb{R}^{3}$, there is no other nonplanar example of circle-foliated minimal surfaces. In $\mathbb{R}^{4}$, the graph $G_{c}$ of $w=c / z$ for real constant $c$ and $\zeta \in \mathbb{C} \backslash\{0\}$ is also foliated by circles. In this paper, we show that every circle-foliated minimal surface in $\mathbb{R}^{n}$ is either a catenoid or Riemann's minimal surface in some 3-dimensional Affine subspace or a graph surface $G_{c}$ in some 4-dimensional Affine subspace. We use the property that $G_{c}$ is circle-foliated to construct circle-foliated minimal surfaces in $S^{4}$ and $H^{4}$.
\end{abstract}

\section{Introduction}

A surface $M \in \mathbb{R}^{n}$ is said to be circle-foliated if there is a one-parameter family of planes whose intersection with $M$ are circles. The catenoid and Riemann's minimal surface are examples of circle-foliated minimal surfaces in $\mathbb{R}^{3}$. Enneper proved that the planes containing the circles of a circle-foliated minimal surface in $\mathbb{R}^{3}$ should be parallel [2] and [7]. Then it is easy to see that the plane, catenoid and Riemann's minimal surface are the only circle-foliated minimal surfaces in $\mathbb{R}^{3}[7]$.

One may consider $\mathbb{R}^{4}$ as $\mathbb{C}^{2}$ with complex coordinates $(z, w)$. For a real constant $c \neq 0$, the graph $G_{c}=\left\{(w, z) \in \mathbb{C}^{2} \mid w z=c\right\}$ is circle-foliated. In fact, the image $g_{r}$ of the circle $\{|z|=r\}$ on the $z$-plane is $\{(z, c / z)|| z \mid=r\}$. Considering $\mathbb{C}^{2}$ as $\mathbb{R}^{4}, g_{r}$ lies on the plane through $(0,0,0,0),\left(1,0,-r^{2}, 0\right)$ and $\left(0,1,0, r^{2}\right)$ (cf. Remark 2). Since $|(z, c / z)|^{2}=r^{2}+c^{2} / r^{2}, g_{r}$ is a circle. Therefore $G_{c}$ is circle-foliated. Since every complex submanifold of a Kaehler manifold is minimal [6], $G_{c}$ is minimal. Moreover, $G_{c}$ is complete, doublyconnected and has finite total curvature $-4 \pi$ with two planar ends, which are asymptotic to the planes $\{z=0\}$ and $\{w=0\}$ (cf. Remark 2). Hoffman and Osserman classified complete simply-connected and doubly-connected minimal surfaces in $\mathbb{R}^{n}$ with total curvature $-4 \pi$ including $G_{c}$ [4]. They showed that

Received February 10, 2014; Revised May 24, 2014.

2010 Mathematics Subject Classification. 53A10, 53C12.

Key words and phrases. circle-foliated surface, minimal surface in $\mathbb{S}^{4}$ and $\mathbb{H}^{4}$.

This work was supported by Hankuk University of Foreign Studies Research Fund. 
such minimal surfaces are foliated by ellipses or circles, and called them as the skew catenoids.

In this paper, we show that every circle-foliated minimal surface in $\mathbb{R}^{n}$ is either a catenoid or a Riemann's minimal surface in a 3-dimensional Affine subspace or the graph surface $G_{c}$ in a 4-dimensional Affine subspace. Therefore there is no counterpart of the Riemann's minimal surface in $\mathbb{R}^{n}$, for $n \geq 4$. We then use this property of $G_{c}$ to construct circle-foliated minimal surfaces in $\mathbb{S}^{4}$ and $\mathbb{H}^{4}$.

\section{Circle-foliated minimal surfaces in $\mathbb{R}^{n}$}

Let $\Sigma$ be a circle-foliated surface in $\mathbb{R}^{n}$. Let $\left\{P_{t}\right\}$ be the one-parameter family of planes on which the circles of the foliation is on. Let $\tilde{P}_{t}$ be the plane parallel to $P_{t}$ and passes through the origin of $\mathbb{R}^{n}$. There is a one-parameter family of orthonormal basis of $\mathbb{R}^{n}$ satisfying Frenet type equations [3].

Theorem A. Let $\left\{\tilde{P}_{t}\right\}$ be a smooth one-parameter family of planes in $\mathbb{R}^{n}$. There is a one-parameter family of orthonormal basis $e_{1}(t), e_{2}(t), \ldots, e_{n}(t)$ of $\mathbb{R}^{n}$ such that $e_{1}(t)$ and $e_{2}(t)$ span $\tilde{P}_{t}$, and the following equations hold

$$
\begin{array}{ll}
e_{i}^{\prime}=\alpha_{i}^{j} e_{j}+\kappa^{i} e_{2+i} & (i, l=1,2) \\
e_{2+i}^{\prime}=-\kappa^{i} e_{i}+\tau_{i}^{l} e_{2+l}+\gamma_{i}^{\lambda} e_{4+\lambda} & (\lambda=1, \ldots, n-4) \\
e_{4+\xi}^{\prime}=-\gamma_{l}^{\xi} e_{2+l}+\beta_{\xi}^{\lambda} e_{4+\lambda} & \left(\alpha_{i}^{j}=-\alpha_{j}^{i}, \tau_{i}^{l}=-\tau_{l}^{i}, \beta_{i}^{\lambda}=-\beta_{\lambda}^{i}\right),
\end{array}
$$

where $\left(\kappa^{1}\right)^{2} \geq\left(\kappa^{2}\right)^{2}$, and ${ }^{\prime}=\frac{d}{d t}$.

Using the above orthonormal basis of $\mathbb{R}^{n}$, we can parameterize a circlefoliated surface by

$$
X(t, \theta)=c(t)+r(t)\left(\cos \theta e_{1}+\sin \theta e_{2}\right),
$$

where $c(t)$ and $r(t)$ are the center and the radius of the circle on $P_{t}$.

Theorem 1. Circle-foliated minimal surface in $\mathbb{R}^{n}$ is either i) a catenoid or a Riemann's minimal surface in 3-dimensional Affine subspace or ii) a graph surface $G_{c}$ (defined in $\S 1$ ) in some 4-dimensional Affine subspace.

To prove the above theorem, we have to show that every circle-foliated minimal surface in $\mathbb{R}^{n}, n \geq 5$, actually lies in a (at most) 4-dimensional Affine subspace. First, let us assume that a circle-foliated surface $X$ lies in $\mathbb{R}^{5}$. (The case of $n \geq 6$ is analogous to the case of $\mathbb{R}^{5}$.) For the simplicity of notations, we write (1) as

$$
\left(\begin{array}{l}
e_{1} \\
e_{2} \\
e_{3} \\
e_{4} \\
e_{5}
\end{array}\right)^{\prime}=\left(\begin{array}{ccccc}
0 & -\beta & -\kappa & 0 & 0 \\
\beta & 0 & 0 & \tau & 0 \\
\kappa & 0 & 0 & -\eta & -\nu \\
0 & -\tau & \eta & 0 & -\mu \\
0 & 0 & \nu & \mu & 0
\end{array}\right)\left(\begin{array}{l}
e_{1} \\
e_{2} \\
e_{3} \\
e_{4} \\
e_{5}
\end{array}\right)
$$


CIRCLE-FOLIATED MINIMAL SURFACES IN 4-DIMENSIONAL SPACE FORMS 1435 Let $c^{\prime}(t)=\sum_{i=1}^{5} \alpha_{i} e_{i}$, where $\alpha_{i}$ 's are smooth functions. Then we have

$$
\begin{aligned}
X_{t}= & \left(\alpha_{1}+r^{\prime} \cos \theta+r \beta \sin \theta\right) e_{1}+\left(\alpha_{2}+r^{\prime} \sin \theta-r \beta \cos \theta\right) e_{2} \\
& +\left(\alpha_{3}-r \kappa \cos \theta\right) e_{3}+\left(\alpha_{4}+r \tau \sin \theta\right) e_{4}+\alpha_{5} e_{5}, \\
X_{\theta}= & -r \sin \theta e_{1}+r \cos \theta e_{2} .
\end{aligned}
$$

Let $N$ be a normal vector of $X$ given by

$$
N=\cos \theta e_{1}+\sin \theta e_{2}+\gamma e_{3}+\delta e_{4}+\rho e_{5} .
$$

Then $\gamma, \delta$ and $\rho$ satisfy

$$
\begin{aligned}
X_{t} \cdot N= & \alpha_{1} \cos \theta+\alpha_{2} \sin \theta+r^{\prime}+\gamma\left(\alpha_{3}-r \kappa \cos \theta\right) \\
& +\delta\left(\alpha_{4}+r \tau \sin \theta\right)+\rho \alpha_{5}=0 .
\end{aligned}
$$

Let $E, F, G$ be the coefficients of the first fundamental form of $X$. Then

$$
\begin{aligned}
E= & \left|X_{t}\right|^{2} \\
= & \sum_{i=1}^{5} \alpha_{i}^{2}+r^{\prime 2}+r^{2} \beta^{2}+2 r^{\prime} \alpha_{1} \cos \theta+2 r^{\prime} \alpha_{2} \sin \theta \\
& +2 r \alpha_{1} \beta \sin \theta-2 r \alpha_{2} \beta \cos \theta-2 r \alpha_{3} \kappa \cos \theta \\
& +r^{2}\left(\kappa^{2}-\tau^{2}\right)(\cos \theta)^{2}+2 r \alpha_{4} \tau \sin \theta+r^{2} \tau^{2}, \\
F= & X_{t} \cdot X_{\theta}=-r \alpha_{1} \sin \theta+r \alpha_{2} \cos \theta-r^{2} \beta, \\
G= & \left|X_{\theta}\right|^{2}=r^{2} .
\end{aligned}
$$

Lemma 1. The surface $X(t, \theta)$ defined by (2) with $\tau \neq 0$ is minimal only if i) $\alpha_{i}=0$ for all $i=1, \ldots, 5$, ii) $\mu=\nu=0$ and iii) $\kappa^{2}=\tau^{2}, \beta \kappa=\tau \eta$ and $\beta \tau=\kappa \tau$. Hence $X(t, \theta)$ lies in a 4-dimensional Affine subspace.

Proof. Let $l=X_{t t} \cdot N, m=X_{t \theta} \cdot N$ and $n=X_{\theta \theta} \cdot N$, where $N$ is given by (4). Since $X(t, \theta)$ is minimal, we must have

$$
\mathcal{H}:=l G+n E-2 m F=0 .
$$

Direct computation shows that

(6)

$$
\begin{aligned}
& r^{2}\left\{\begin{array}{c}
\alpha_{1}^{\prime} \cos \theta+r^{\prime \prime}+\alpha_{2} \beta \cos \theta-\alpha_{1} \beta \sin \theta+\alpha_{2}^{\prime} \sin \theta-r \beta^{2} \\
+\alpha_{3} \kappa \cos \theta-r \kappa^{2}(\cos \theta)^{2}-\alpha_{4} \tau \sin \theta-r \tau^{2}(\sin \theta)^{2} \\
+\gamma\left(\begin{array}{l}
\alpha_{3}^{\prime}-2 r^{\prime} \kappa \cos \theta-\alpha_{1} \kappa-r \kappa^{\prime} \cos \theta \\
-r \beta \kappa \sin \theta+\alpha_{4} \eta+r \tau \eta \sin \theta+\alpha_{5} \nu
\end{array}\right) \\
+\delta\left(\begin{array}{l}
\alpha_{4}^{\prime}+2 r^{\prime} \tau \sin \theta+\alpha_{2} \tau+r \tau^{\prime} \sin \theta \\
-r \beta \tau \cos \theta-\alpha_{3} \eta+r \kappa \eta \cos \theta+\alpha_{5} \mu
\end{array}\right) \\
+\rho\left(\alpha_{5}^{\prime}+r \kappa \nu \cos \theta-\alpha_{3} \nu-\alpha_{4} \mu-r \tau \mu \sin \theta\right)
\end{array}\right\} \\
& -r\left\{\begin{array}{l}
\sum_{i=1}^{5} \alpha_{i}^{2}+r^{\prime 2}+r^{2} \beta^{2}+2 r^{\prime} \alpha_{1} \cos \theta+2 r^{\prime} \alpha_{2} \sin \theta \\
+2 r \alpha_{1} \beta \sin \theta-2 r \alpha_{2} \beta \cos \theta-2 r \alpha_{3} \kappa \cos \theta \\
+r^{2}\left(\kappa^{2}-\tau^{2}\right)(\cos \theta)^{2}+2 r \alpha_{4} \tau \sin \theta+r^{2} \tau^{2}
\end{array}\right\} \\
& -2(r \beta+r \gamma \kappa \sin \theta+r \delta \tau \cos \theta)\left(-r \alpha_{1} \sin \theta+r \alpha_{2} \cos \theta-r^{2} \beta\right) \text {. }
\end{aligned}
$$


Since $\gamma, \delta$ and $\rho$ satisfy (5), we first let $\gamma=-\left(\alpha_{1} \cos \theta+\alpha_{2} \sin \theta+r^{\prime}\right) /\left(\alpha_{3}-\right.$ $r \kappa \cos \theta), \delta=\rho=0$ and $\mathcal{S}:=\mathcal{H}\left(\alpha_{3}-r \kappa \cos \theta\right)$. Then the coefficients of $\cos (3 \theta)$ and $\sin (3 \theta)$ of $\mathcal{S}$ are $r^{2} \kappa\left(r^{2}\left(\kappa^{2}-\tau^{2}\right)+\alpha_{1}^{2}-\alpha_{2}^{2}\right) / 2$ and $r^{2} \kappa \alpha_{1} \alpha_{2}$ respectively. Since these should be equal to zero and $\kappa^{2} \geq \tau^{2}$, we necessarily have $\alpha_{1}=0$.

Let $\delta=-\left(\alpha_{2} \sin \theta+r^{\prime}\right) /\left(\alpha_{4}+r \tau \sin \theta\right), \gamma=\rho=0$ and $\mathcal{T}:=\mathcal{H}\left(\alpha_{4}+r \tau \cos \theta\right)$. The coefficients of $\cos (2 \theta)$ of $\mathcal{S}$ and $\sin (2 \theta)$ of $\mathcal{T}$ are $r^{3}\left(-5 \alpha_{3} \kappa^{2}+2 \alpha_{3} \tau^{2}+\alpha_{2} \tau \eta\right)$ and $\left(3 r^{3} \alpha_{3} \kappa \tau-r^{3} \alpha_{2} \kappa \eta\right) / 2$ respectively, which are equal to 0 . Hence we have either $\kappa^{2}-\tau^{2}=0$ or $\alpha_{3}=0$. On the other hand, the coefficient of $\cos (3 \theta)$ of $\mathcal{T}$ is $-r^{4} \tau\left(\kappa^{2}-\tau^{2}\right)$, which implies that $\kappa^{2}=\tau^{2}$. Then we have $\alpha_{2}=0$, and since we assumed that $\tau^{2}>0$, we also have $\alpha_{3}=0$. Substituting these into $\mathcal{S}$, we have $\alpha_{4}=0$ from the coefficient of $\sin (2 \theta)$.

Suppose that $\alpha_{5} \neq 0$, and let $\gamma=\delta=0$ and $\rho=-r^{\prime} / \alpha_{5}$. Then $\mathcal{H}$ becomes

$$
\begin{aligned}
& r^{2}\left[r^{\prime \prime}-2 r \kappa^{2}(\cos \theta)^{2}-2 r \tau^{2}(\sin \theta)^{2}-\frac{r^{\prime}}{\alpha_{5}}\left(\alpha_{5}^{\prime}+r \kappa \nu \cos \theta-r \tau \nu \sin \theta\right)\right] \\
& -r\left(\alpha_{5}^{2}+{r^{\prime}}^{2}\right) .
\end{aligned}
$$

Therefore we have $\kappa=\tau=0$, which contradicts the assumption $\kappa, \tau \neq 0$. From (5), (6) and $\alpha_{5}=0$, it follows that $\mu=\nu=0$. This completes the proofs of i) and ii).

From the coefficients of $\sin \theta$ of $\mathcal{S}$ and $\cos \theta$ of $\mathcal{T}$, we have

$$
\beta \kappa=\tau \eta
$$

and

$$
\beta \tau=\kappa \eta .
$$

Since $\mu=\nu=0$ and $\alpha_{i}=0$ for all $i=1, \ldots, 5$, the surface $X(t, \theta)$ lies in a 4-dimensional Affine subspace.

Remark 1 . When $n \geq 6$ and $X(t, \theta)$ is minimal, it is easy to see in the above proof that $\alpha_{k}=0$ for $k \geq 5$ and $\gamma_{i}^{\lambda}=0$ and $\beta_{\xi}^{\lambda}=0$. Hence $X(t, \theta)$ should lie in a 4-dimensional Affine subspace.

Lemma 2. If the surface $X(t, \theta)$ defined by (2) is minimal with $\tau \equiv 0$, then the planes $\tilde{P}$ lie in some 3 -dimensional Affine subspace.

Proof. When $\tau \equiv 0$, we consider two cases; $\alpha_{4} \equiv 0$ or $\alpha_{4} \not \equiv 0$. First of all, we have $\alpha_{1}=0$ as in the proof of the above lemma. If $\tau \equiv 0$ and $\alpha_{4} \not \equiv 0$, then we let $\gamma=\rho=0$ and $\delta=-\left(\alpha_{2} \sin \theta+r^{\prime}\right) / \alpha_{4}$. The coefficient of $\cos (2 \theta)$ of $\mathcal{H}$ is $-2 r^{3} \kappa^{3}$. Since this must be 0 , we have $\kappa \equiv 0$. Then $\eta, \nu$ and $\mu$ can be chosen to be zero, and $\tilde{p}$ are parallel planes in a 3-dimensional Affine subspace.

If $\alpha_{4} \equiv 0$, then $\mathcal{H}$ is independent of the choice of $\delta$. Hence we have $\eta \equiv 0$ and $\alpha_{5} \mu \equiv 0$. If $\alpha_{5} \equiv 0$, then we should have $\nu \equiv 0$. From (3), $e_{4}$ and $e_{5}$ are independent of $e_{1}, e_{2}$ and $e_{3}$, and $\tilde{p}$ lie in a 3-dimensional Affine subspace. If $\alpha_{5} \neq \equiv 0$, let $\gamma=\delta=0$ and $\rho=-\left(\alpha_{2} \sin \theta+r^{\prime}\right) / \alpha_{5}$. Then the coefficient of $\cos (2 \theta)$ of $\alpha_{5} \mathcal{H}$ is $-2 r^{3} \kappa$, which should be 0 . Therefore $e_{1}$ and $e_{2}$ are independent of $e_{3}$, $e_{4}$ and $e_{5}$, and $\tilde{p}$ lie in a 3 -dimensional Affine subspace. 
Lemma 3. The circle-foliated minimal surfaces in $\mathbb{R}^{4}$ of Lemma 1 is the graph $G_{c}$ for some real $c$.

Proof. From (7), (8) and $\kappa^{2}=\tau^{2}$, we see that $\beta^{2}=\kappa^{2}=\tau^{2}=\eta^{2}$. Suppose that $\beta=\kappa$ and $\tau=\eta$ (the case $\beta=-\kappa$ and $\tau=-\eta$ can be dealt with in the same way). It follows that $\left(e_{2}+e_{3}\right)^{\prime}=0$ and $\left(e_{1} \pm e_{4}\right)^{\prime}=0$ (depending on the sign of $\kappa / \tau)$. We may suppose that $e_{1}+e_{4}=(0,0,0, \sqrt{2})$ and $e_{2}+e_{3}=(0,0, \sqrt{2}, 0)$. Then we have

$$
\begin{aligned}
& e_{1}=\frac{1}{\sqrt{2}}(\cos \psi(t), \sin \psi(t), 0,1), \\
& e_{2}=\frac{1}{\sqrt{2}}(\cos \phi(t), \sin \phi(t), 1,0), \\
& e_{3}=\frac{1}{\sqrt{2}}(-\cos \phi(t),-\sin \phi(t), 1,0), \\
& e_{4}=\frac{1}{\sqrt{2}}(-\cos \psi(t),-\sin \psi(t), 0,1) .
\end{aligned}
$$

From $e_{1}^{\prime}=-\beta e_{2}+\beta e_{3}$, we see that $2 \beta= \pm \psi^{\prime}$. If $\psi^{\prime}=2 \beta$, then we have $\psi=\pi / 2+\phi$. Moreover $e_{3}^{\prime}=-\beta e_{1}+\tau e_{4}$ implies that $\beta=\eta$. Similarly, when $\psi^{\prime}=-2 \beta$, we have $\kappa=\eta$. Therefore we may assume that $\beta=\kappa=\tau=\eta=1$. Then direct computation shows that

$$
\mathcal{H}=r\left(r r^{\prime \prime}-3 r^{2}-2 r^{2}\right)
$$

for the normals of $X(t, \theta)$ corresponding to the cases i) $\gamma=-r^{\prime} / r \cos \theta, \delta=0$ and ii) $\gamma=0, \delta=r^{\prime} / r \sin \theta$. Hence $r$ satisfies

$$
r r^{\prime \prime}-3 r^{2}-2 r^{2}=0 .
$$

The solution of (9) is $r=C_{1}\left(\cos \left(2 t+C_{2}\right)\right)^{-1 / 2}$, where $C_{1}$ and $C_{2}$ are constants.

We may let $C_{1}=c$ and $C_{2}=0$ and $-\pi / 4<t<\pi / 4$. Let $A$ be the $4 \times 4$ orthogonal matrix given by

$$
A=\frac{1}{\sqrt{2}}\left(\begin{array}{cccc}
1 & 0 & 0 & 1 \\
0 & -1 & 1 & 0 \\
0 & 1 & 1 & 0 \\
-1 & 0 & 0 & 1
\end{array}\right)
$$

Then $\tilde{X}(t, \theta)=A \circ X(t, \theta)$ represents the graph

$$
G_{c}=\left\{\left(\zeta, \frac{c}{\zeta}\right) \mid \zeta \in \mathbb{C}-\{0\}\right\} .
$$

Remark 2. i) The parametrization of $G_{c}$ is given by

$$
\tilde{X}(t, \theta)=\left(r \cos \theta, r \sin \theta, \frac{c}{r} \cos \theta,-\frac{c}{r} \sin \theta\right) .
$$

Clearly, $G_{c}$ has two ends that are asymptotic to the planes $\{(w, z) \mid w=0\}$ and $\{(w, z) \mid z=0\}$. 
ii) Let $g_{r}=\left(r \cos \theta, r \sin \theta, \frac{c}{r} \cos \theta,-\frac{c}{r} \sin \theta\right)$ be the circle on $G_{c}$ for fixed $r$. The geodesic curvature of $g_{r}$ is

$$
\kappa_{g}=\frac{r\left|c^{2}-r^{4}\right|}{\left(c^{2}+r^{4}\right)^{3 / 2}} .
$$

Hence we have

$$
\int_{g_{r}} \kappa_{g} d s=2 \pi \frac{\left|c^{2}-r^{4}\right|}{c^{2}+r^{4}}
$$

Since $G_{c}$ is doubly-connected, Gauss-Bonnet theorem implies that

$$
\int_{G_{c}} K d A=-\lim _{r \rightarrow 0} \int_{g_{r}} \kappa_{g} d s-\lim _{r \rightarrow \infty} \int_{g_{r}} \kappa_{g} d s=-4 \pi .
$$

\section{Circle-foliated minimal surfaces in $\mathbb{S}^{4}$ and $\mathbb{H}^{4}$}

To construct a circle-foliated minimal surface in $\mathbb{S}^{4}$, we consider $\mathbb{R}^{4}$ with the conformal metric $d s_{s}^{2}=d s_{0}^{2} /((1+\langle x, x\rangle) / 2)^{2}$, where $d s_{0}^{2}$ is the Euclidean metric of $\mathbb{R}^{4}$ and $\langle$,$\rangle is the Euclidean inner product. Let H_{s}$ and $H_{0}$ be the mean curvatures of a surface $M$ in $\mathbb{R}^{4}$ with respect to the metrics $d s_{s}^{2}$ and $d s_{0}^{2}$ respectively with respect to fixed Euclidean normal $N$ satisfying (4). We have

$$
H_{s}=\frac{1+\langle x, x\rangle}{2|N|} H_{0}+\left\langle x, \frac{N}{|N|}\right\rangle .
$$

Similarly, to construct a circle-foliated minimal surfaces in $\mathbb{H}^{4}$, we equip the conformal metric $d s_{h}^{2}=d s_{0}^{2} /((1-\langle x, x\rangle) / 2)^{2}$ on the unit ball $B(O, 1)$ of $\mathbb{R}^{4}$. Then the mean curvature $H_{h}$ of a surface $M$ in $B(O, 1)$ with respect to $d s_{h}^{2}$ satisfies

$$
H_{h}=\frac{1-\langle x, x\rangle}{2|N|} H_{0}-\left\langle x, \frac{N}{|N|}\right\rangle .
$$

Examples of circle-foliated minimal surfaces in $\mathbb{S}^{4}$ and $\mathbb{H}^{4}$. Let $e_{1}, e_{2}$ be defined as in the proof of Theorem 3:

$$
\begin{aligned}
& e_{1}=\frac{1}{\sqrt{2}}(-\sin 2 t, \cos 2 t, 0,1), \\
& e_{2}=\frac{1}{\sqrt{2}}(\cos 2 t, \sin 2 t, 1,0) .
\end{aligned}
$$

The mean curvature of the circle-foliated surface

$$
X(t, \theta)=r(t)\left(\cos \theta e_{1}+\sin \theta e_{2}\right)
$$

satisfies

$$
H_{0}|N|=\frac{r\left(r r^{\prime \prime}-3{r^{\prime}}^{2}-2 r^{2}\right)}{2 r^{2}\left(r^{2}+r^{\prime 2}\right)} .
$$


Hence as a surface in $\mathbb{S}^{4}, X(t, \theta)$ has mean curvature

$$
H_{s}|N|=\frac{1+r^{2}}{2} \cdot \frac{r\left(r r^{\prime \prime}-3{r^{\prime}}^{2}-2 r^{2}\right)}{2 r^{2}\left(r^{2}+{r^{\prime}}^{2}\right)}+r
$$

for all normal direction. Therefore the circles centered at the origin on the planes spanned by $e_{1}(t), e_{2}(t)$ with radius function $r(t)$ satisfying

$$
\frac{1+r^{2}}{2} \cdot \frac{r r^{\prime \prime}-3 r^{\prime 2}-2 r^{2}}{2 r^{2}\left(r^{2}+r^{\prime 2}\right)}+1=0
$$

define a circle-foliated minimal surface in $\mathbb{S}^{4}$.

Lemma 4. Solution of (12) with the initial conditions

$$
r(0)=a^{2}>0 \text { and } r^{\prime}(0)=0
$$

is periodic.

Proof. Note that if $r(t)$ is a solution of (12), then $r(-t)$ is also a solution of (12). Hence each solution $r$ of (12) is an even function. Moreover if $r^{\prime}\left(t_{1}\right)=0$, then $r\left(t_{1}+t\right)=r\left(t_{1}-t\right)$. Therefore it suffices to show that $r^{\prime}\left(t_{1}\right)=0$ for some $t_{1}>0$.

Suppose that $r^{\prime}$ does not vanish except for $t=0$, therefore, $r^{\prime}(t)>0$ for all $t>0$. From (12), we have

$$
r^{\prime \prime}=\frac{\left(3-r^{2}\right) r^{2}+2 r^{2}\left(1-r^{2}\right)}{r\left(1+r^{2}\right)} .
$$

We may assume that $a<1$. Then we have $r^{\prime \prime}(0)>0$ and $r^{\prime}(t)>0$ for $t$ close to 0 . If $r$ is not bounded, then $r^{\prime \prime} \rightarrow-\infty$ as $t \rightarrow \infty$ by (13). Then $r^{\prime} \rightarrow-\infty$, which contradicts $r^{\prime}(t)>0$ for all $t>0$.

If $r$ is bounded, then $r^{\prime \prime} \rightarrow 0$ and $r^{\prime} \rightarrow 0$ as $t \rightarrow \infty$. From (13) and the fact that $r^{\prime \prime} \rightarrow 0$ as $t \rightarrow \infty$, it follows that $r \nearrow 1$ as $t \rightarrow \infty$. From (13), we have $r^{\prime \prime}(t)>0$ for all $t>0$. On the other hand, since $r$ is bounded and increasing, we should have $r^{\prime \prime}(t)<0$ for sufficiently large $t$. Hence we conclude that $r^{\prime}\left(t_{1}\right)=0$ for some $t_{1}$ and $r$ is periodic.

To estimate the period of (13), we use the integrating factor to obtain a first integral

$$
\frac{\left(1+r^{2}\right)^{4}}{r^{6}}\left(r^{\prime}\right)^{2}+\left(\frac{1}{r^{4}}+\frac{4}{r^{2}}+4 r^{2}+r^{4}\right)=C
$$

or

$$
\left(r+\frac{1}{r}\right)^{4}\left(\frac{r^{\prime}}{r}\right)^{2}+\left(r+\frac{1}{r}\right)^{4}=C .
$$


We suppose that $r^{\prime}(0)=0$ and the minimum value $r_{\min }$ is attained at $t=0$, that is, $r_{\min }=r(0)$. Then

$$
C=\left(r_{\min }+\frac{1}{r_{\min }}\right)^{4}
$$

Let $r_{\max }$ be the maximum value of $r$ and $r_{\max }=r\left(t_{\max }\right)$ so that the period of $r$ is $2 t_{\max }$. Then have

$$
r_{\min }=\frac{1}{r_{\max }}
$$

Since $r$ is strictly increasing on $\left(0, t_{\max }\right)$, we consider the inverse function $t=t(r)$ of $r(t)$. From (14), we have

$$
t^{\prime 2}=\frac{1}{r^{2}} \frac{\left(r^{2}+1\right)^{4}}{C r^{4}-\left(r^{2}+1\right)^{4}} .
$$

Then

$$
t_{\max }=\int_{r_{\min }}^{r_{\max }} \frac{1}{r} \frac{\left(r^{2}+1\right)^{2}}{\sqrt{C r^{4}-\left(r^{2}+1\right)^{4}}} d r .
$$

Since $1 / r$ also satisfies (12), we have

$$
t_{\max }=2 \int_{r_{\min }}^{1} \frac{1}{r} \frac{\left(r^{2}+1\right)^{2}}{\sqrt{C r^{4}-\left(r^{2}+1\right)^{4}}} d r .
$$

Substituting $R=r^{2}$, we get

$$
t_{\max }=2 \int_{r_{\min }^{2}}^{1} \frac{1}{2 R} \frac{(R+1)^{2}}{\sqrt{C R^{2}-(R+1)^{4}}} d R .
$$

For convenience, we let $c^{2}=C$ with $c \geq 4, p=\sqrt{(c+4) / c}$ and $k^{2}=(c+$ $4) /(c-4)$.

Substituting $R=(\rho-p) /(\rho+p)$, we have

$$
\begin{aligned}
\frac{d R}{\sqrt{c^{2} R^{2}-(R+1)^{4}}} & =\frac{2 p d \rho}{\sqrt{(c+4)\left(\rho^{2}-1\right)\left((c-4) \rho^{2}-(c+4)\right)}} \\
& =\frac{2}{\sqrt{c(c-4)}} \frac{d \rho}{\sqrt{\left(\rho^{2}-1\right)\left(\rho^{2}-k^{2}\right)}} .
\end{aligned}
$$

Then (15) becomes

$$
t_{\max }=\frac{8}{\sqrt{c(c-4)}} \int_{\rho_{0}}^{\infty} \frac{\rho^{2} d \rho}{\left(\rho^{2}-p^{2}\right) \sqrt{\left(\rho^{2}-1\right)\left(\rho^{2}-k^{2}\right)}},
$$

where $\rho_{0}=p\left(1+r_{\min }^{2}\right) /\left(1-r_{\min }^{2}\right)=k$. 
Substituting $\tau=k / \rho$, we get

$$
\begin{aligned}
t_{\max } & =\frac{8}{\sqrt{c(c+4)}} \int_{0}^{1} \frac{d \tau}{\left(1-\left(\frac{p}{k}\right)^{2} \tau^{2}\right) \sqrt{\left(1-\tau^{2}\right)\left(1-\frac{\tau^{2}}{k^{2}}\right)}} \\
& =\frac{8}{\sqrt{c(c+4)}} \Pi\left(\frac{c-4}{c} \mid \frac{c-4}{c+4}\right),
\end{aligned}
$$

where $\Pi((c-4) / c \mid(c-4) /(c+4))$ is the complete elliptic integral of the third kind. For the following lemma, we introduce the elliptic integral of the 1st kind $F(\phi, \alpha)$ and the elliptic integral of the 2nd kind $E(\phi, \alpha)$ :

$$
\begin{aligned}
& F(\phi, \alpha)=\int_{0}^{\phi} \frac{d \theta}{\sqrt{1-\sin ^{2} \alpha \sin ^{2} \theta}}, \\
& E(\phi, \alpha)=\int_{0}^{\phi} \sqrt{1-\sin ^{2} \alpha \sin ^{2} \theta} d \theta .
\end{aligned}
$$

Moreover, $K(\alpha)=F(\pi / 2, \alpha)$ and $E(\alpha)=E(\pi / 2, \alpha)$ are the complete elliptic integrals of the first and second kinds respectively. Letting $k=\sin \alpha$, we also let $E(k)=E(\pi / 2 ; k), K(k)=F(\pi / 2 ; k)$.

Note that $c^{2}=\left(r_{\min }+1 / r_{\min }\right)^{2}$. If $c \rightarrow 4$ or $r_{\min } \rightarrow 1$, then $t_{\max } \rightarrow \pi / \sqrt{2}$. In this case, we have $r \equiv 1$ and the resulting minimal surface is a torus.

Lemma 5. As a function of $c \geq 4, t_{\max }$ is decreasing and satisfies

$$
\lim _{c \rightarrow \infty} t_{\max }=\frac{\pi}{2} \text {. }
$$

Hence the period of the solution of (12) is between $\pi$ and $\sqrt{2} \pi$.

Proof. Straightforward computation shows that

$$
\frac{d}{d c}\left(\frac{8}{\sqrt{c(c+4)}} \Pi\left(\frac{c-4}{c} \mid \frac{c-4}{c+4}\right)\right)=\frac{E\left(\frac{c-4}{c+4}\right)-K\left(\frac{c-4}{c+4}\right)}{2(c-4) \sqrt{c(c+4)}}
$$

Since

$$
E\left(\frac{c-4}{c+4}\right)-K\left(\frac{c-4}{c+4}\right)<0
$$

$t_{\max }$ is a decreasing function of $c$.

Let $\alpha=\sin ^{-1} \sqrt{(c-4) /(c+4)}$ with $0<\alpha<\pi / 2$ and let $\nu=(c-4) / c$. According to $[1]$, the integral $\Pi((c-4) / c ; \pi / 2,(c-4) /(c+4))=\Pi(\nu ; \pi / 2, \alpha)$ belongs to the circular case with $\sin ^{2} \alpha<\nu<1$, and

$$
\Pi(\nu ; \pi / 2, \alpha)=K(\alpha)+\frac{\pi}{2} \delta_{2}\left(1-\Lambda_{0}(\phi, \alpha)\right),
$$

where $\Lambda_{0}$ is the Heuman's Lambda function satisfying

$$
\Lambda_{0}(\phi, \alpha)=\frac{2}{\pi}[K(\alpha)(E(\phi, \pi / 2-\alpha)-F(\phi, \pi / 2-\alpha))+E(\alpha) F(\phi, \pi / 2-\alpha)],
$$


and

$$
\begin{gathered}
\delta_{2}=\sqrt{\nu /(1-\nu)\left(\nu-\sin ^{2} \alpha\right)}=\sqrt{c(c+4)} / 4, \\
\phi=\sin ^{-1} \sqrt{(1-\nu) / \cos ^{2} \alpha}=\sin ^{-1} \sqrt{(c+4) / 2 c} .
\end{gathered}
$$

Therefore $\phi \rightarrow \pi / 4$ and $\alpha \rightarrow \pi / 2$ as $c \rightarrow \infty$.

Clearly,

$$
\lim _{c \rightarrow \infty}(E(\phi, \pi / 2-\alpha)-F(\phi, \pi / 2-\alpha))=-\lim _{c \rightarrow \infty} \int_{0}^{\phi} \frac{\cos ^{2} \alpha \sin ^{2} \theta}{\sqrt{1-\cos ^{2} \alpha \sin ^{2} \theta}} d \theta .
$$

We note that $\cos \alpha=\sqrt{8 /(c+4)}$ and that $\lim _{\alpha \nearrow \pi / 2} \cos \alpha K(\alpha)=0$ (cf. Lemma 8 of [5]). Then

$$
\lim _{c \rightarrow \infty} t_{\max }=\lim _{c \rightarrow \infty} \frac{8}{\sqrt{c(c+4)}} \Pi(\nu ; \pi / 2, \alpha)=\frac{\pi}{2} .
$$

Theorem 2. The circle-foliated surface given by (11) with $e_{1}, e_{2}$ satisfying (10) and $r$ satisfying (12) defines a one-parameter family of circle-foliated minimal surfaces in $\mathbb{S}^{4}$. Moreover, the radius function $r$ is periodic with the period between $\pi$ and $\sqrt{2} \pi$. Hence there are infinitely many circle-foliated immersed minimal tori in $\mathbb{S}^{4}$.

In $\mathbb{H}^{4}$, we let $e_{1}, e_{2}$ and $X(t, \theta)$ be as in (10) and (11). Then the mean curvature of $X(t, \theta)$ with respect to $d s_{h}^{2}$ satisfies

$$
H_{h}|N|=\frac{1-r^{2}}{2} \cdot \frac{r\left(r r^{\prime \prime}-3{r^{\prime}}^{2}-2 r^{2}\right)}{2 r^{2}\left(r^{2}+{r^{\prime}}^{2}\right)}-r
$$

with $N$ satisfying (4). Hence if $X(t, \theta)$ is minimal, then $r$ satisfies

$$
\frac{1-r^{2}}{2} \cdot \frac{r r^{\prime \prime}-3 r^{\prime 2}-2 r^{2}}{2 r^{2}\left(r^{2}+r^{\prime 2}\right)}-1=0
$$

We note that $r^{\prime \prime}$ blows up as $r \rightarrow 1$, when $X(t, \theta)$ approaches the ideal boundary of $\mathbb{H}^{4}$. For each initial condition $r(0)=b^{2}<1, r^{\prime}(0)=0$ of $(17), X(t, \theta)$ gives a complete circle-foliated minimal surface in $\mathbb{H}^{4}$.

Theorem 3. The parametrization (11) with $e_{1}, e_{2}$ satisfying (10) and $r$ satisfying (17) gives a one-parameter family of circle-foliated minimal surfaces in $\mathbb{H}^{4}$.

\section{References}

[1] M. Abramowitz and I. A. Stegun, Handbook of mathematical functions with formulas, graphs, and mathematical tables, National Bureau of Standards Applied Mathematics Series 55, Government Printing Office, 1964.

[2] A. Enneper, Die cyklischen flächen, Z. Math Phys. 14 (1869), 393-421.

[3] H. Frank and O. Giering, Verallgemeinerte Regelflächen, Math. Z. 150 (1976), no. 3, $261-271$. 
CIRCLE-FOLIATED MINIMAL SURFACES IN 4-DIMENSIONAL SPACE FORMS 1443

[4] D. Hoffman and R. Osserman, The geometry of the generalized Gauss map, Mem. Amer. Math. Soc. 28 (1980), no. 236, 105 pp.

[5] R. Hynd, S. Park, and J. McCuan, Symmetric surfaces of constant mean curvature in $\mathbb{S}^{3}$, Pacific J. Math. 241 (2009), no. 1, 63-115.

[6] H. Lawson, Complete minimal surfaces in $\mathbb{S}^{3}$, Ann. Math. 92 (1970), no. 3, 335-374.

[7] J. Nitsche, Lectures on Minimal Surfaces, Cambridge Univ. Press, Cambridge, 1989.

Major in Mathematics

Graduate School of Education

Hankuk University of Foreign Studies

SeOul 130-791, Korea

E-mail address: sunghopark@hufs.ac.kr 